\title{
Sostenibilidad de las unidades de producción del cultivo de amaranto (Amaranthus caudatus L.)
}

\section{Sustainability of the production units of the amaranto crop (Amaranthus caudatus L.)}

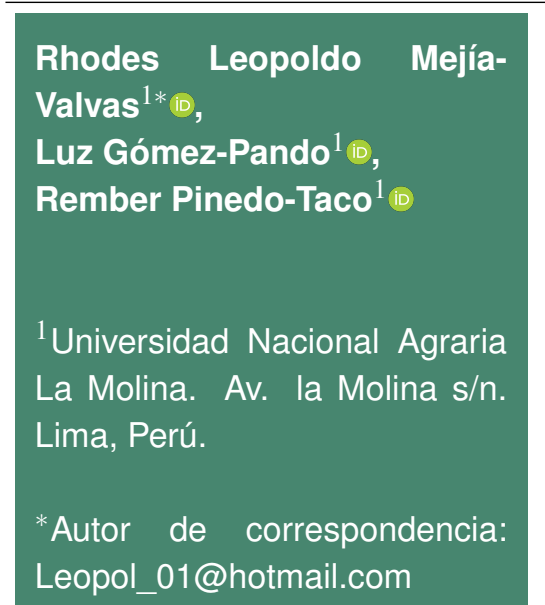

Nota científica

Recibida: 29 de noviembre de 2019

Aceptada: 26 de mayo de 2020

Como citar: Mejía-Valvas RL, Gómez-Pando L, Pinedo-Taco R (2020) Sostenibilidad de las unidades de producción del cultivo de amaranto (Amaranthus caudatus L.). Ecosistemas y Recursos Agropecuarios 7(2): e2483. DOI: 10.19136/era.a7n2.2483
RESUMEN. El amaranto (Amaranthus caudatus L.), es un cultivo marginal en los sistemas agrícolas del Perú. El objetivo de la investigación fue determinar la sostenibilidad de las unidades de producción de amaranto del departamento de Ancash. En una muestra estratificada de 115 agricultores de cinco comunidades se aplicaron encuestas con preguntas estructuradas para conocer la situación actual y las tendencias de la producción de amaranto en las dimensiones económicas, ambientales y sociales. Los datos se estandarizaron para cada indicador mediante transformación a una escala de 1 a 5 ; siendo 5 el mayor valor de sostenibilidad y 1 el más bajo. Se consideró que una unidad productiva de amaranto es sostenible si es mayor a 3, denominado umbral mínimo de sostenibilidad. El índice de sustentabilidad general para las unidades de producción de amaranto fue no sostenible al no alcanzar la escala mínima de 3 en el análisis multidimensional.

Palabras clave: Análisis multivariado, indicador, seguridad alimentaria, umbral de sostenibilidad.

ABSTRACT. Amaranth (Amaranthus caudatus L.), is considered a marginal crop in Peru agricultural systems. The aim of the research was to determine the sustainability of the amaranth production units in the department of Ancash. Surveys with structured questions were applied to a stratified sample of 115 farmers from five communities to find out the current situation and trends in amaranth production in the economic, environmental and social dimensions. The data were standardized for each indicator by transformation on a scale of 1 to $5 ; 5$ being the highest sustainability value and 1 the lowest. A productive unit of amaranth was considered to be sustainable if it is greater than 3 , called the minimum sustainability threshold. The general sustainability index for the amaranth production units was unsustainable as it did not reach the minimum scale of 3 in the multidimensional analysis.

Key words: Multivariate analysis, indicator, food security, sustainability threshold. 


\section{INTRODUCCIÓN}

Las especies de amaranto (Amaranthus caudatus L.), son cultivos agrícolas de gran interés en muchas regiones del mundo por su alto valor nutritivo (Vera y Lerma 2015); según Matías et al. (2018), es originario de los andes de Sudamérica. El cultivo se conoce como kiwicha en Perú, el cual tiene posibilidades y perspectivas técnicas de desarrollo por las características agroclimáticas, edáficas y tecnológicas favorables (Vera y Lerma 2015). Pero aún con estas ventajas, la superficie cultivada no es significativa; siendo las principales zonas productoras los departamentos de Apurímac, cusco, Ancash, Ayacucho, Huancavelica y la Libertad, con una superficie cultivada de $1480 \mathrm{ha}^{-1}$, con rendimiento promedio de $1885 \mathrm{~kg} \mathrm{ha}^{-1}$. Para departamento de Ancash, en 1990 la superficie cultivada fue de188 ha, con rendimiento $676 \mathrm{~kg} \mathrm{ha}^{-1}$ y producción de $127 \mathrm{t}$; mientras que en el 2014 fue de 370 ha con rendimiento promedio de $1638 \mathrm{~kg} \mathrm{ha}^{-1}$ (MINAGRI 2017).

La mayor parte de los actuales sistemas de producción de amaranto y otros granos andinos en Perú, se cultivan de forma marginal y no se consideran en el Plan Nacional de Cultivos (MINAGRI 2018b). Aun cuando es un cultivo autóctono y adaptado a las condiciones ecológicas de los andes, es un cultivo subutilizado por falta de fomento, asistencia técnica e investigación (Bravo et al. 2010, Vera y Lerma 2015). En el actual contexto, se desconocen los niveles de sostenibilidad del cultivo en los aspectos económicos, ambientales, sociales e institucionales. De acuerdo con Sarandón (2002) y Sarandón y Flores (2014), la agricultura sustentable debe ser productiva, viable, adecuada, cultural y socialmente aceptable. En la actualidad, hay propuestas metodológicas que demuestran la utilidad para medir o cuantificar la rentabilidad, competitividad, niveles de perturbación de los agroecosistemas, prácticas de intensificación agrícola, prácticas conservacionistas, grado de satisfacción de necesidades básicas y bienestar de los agricultores, entre otras (Sarandón 2002, Silva y Ramírez 2017, Pinedo et al. 2018). Por consiguiente, para los sistemas agrícolas de las zonas altoandinas, los niveles de sostenibilidad se puede medir con indicadores, de acuerdo a la tipología y sistemas de producción (Pinedo et al. 2017, Pinedo et al. 2018). Al respecto, Sarandón (2002) plantea una propuesta metodológica multivariada para definir un índice general de sostenibilidad con el uso de indicadores económicos, ambientales y sociales; esta metodología permite identificar los puntos relevantes y críticos de los sistemas de producción, además de establecer un umbral mínimo de sostenibilidad de acuerdo con una escala previamente establecida. Con respecto, a la sustentabilidad del cultivo, aún no hay acuerdo en la forma de cuantificar la toma de decisiones prácticas que encaucen el desarrollo sostenible (Castelán et al. 2014, Silva y Ramírez 2017). Por lo anterior, el objetivo del presente estudio fue evaluar la sustentabilidad de producción de amaranto en cinco comunidades de Ancash.

\section{MATERIALES Y MÉTODOS}

La investigación se realizó en las provincias de Huaylas, Yungay y Carhuaz del departamento de Ancash (Tabla 1). La información se obtuvo de encuestas aplicadas a los productores de amaranto con preguntas estructuradas (Rocha et al. 2016, Pinedo et al. 2017, Pinedo et al. 2018). De una población de 448 productores de amaranto se tomó una muestra al azar de 115 productores que participaron, proporcionando información sobre la situación actual y las tendencias en las dimensiones: económicas, ambientales y sociales, para definir la sostenibilidad del cultivo (Silva y Ramírez 2017, Pinedo et al. 2018). Como en la zona de estudio las comunidades presentan diferente número de unidades de muestreo, la distribución se realizó de forma proporcional (Márquez 2015, Pinedo et al. 2018). El análisis de sostenibilidad se realizó con enfoque multidimensional en base a la propuesta metodológica de Sarandón (2002) y Sarandón et al. (2006). Se utilizaron 10 indicadores y 24 sub-indicadores, que se agruparon en las dimensiones económica, ambiental y social (Tabla 2). Considerando que los indicadores seleccionados difieren entre las unidades de producción, lo que dificulta la comparación entre ellas, se construyó una 
Tabla 1. Muestra calculada proporcionalmente por comunidad estudiada en las provincias de Huaylas, Yungay y Carhuaz del departamento de Ancash.

\begin{tabular}{lllcccc}
\hline Provincia & Distrito & Comunidad & Altitud & $\begin{array}{c}\text { Población por } \\
\text { comunidad (N1) }\end{array}$ & $\begin{array}{c}\text { Peso proporcional de la } \\
\text { muestra }(\mathrm{Wi}=\mathrm{Ni} / \mathrm{N})\end{array}$ & $\begin{array}{c}\text { Muestra(n) } \\
\mathrm{N}=\mathrm{NWi}\end{array}$ \\
\hline Huaylas & Mato & Mato & 2234 & $\mathrm{~N} 1=130$ & 0.20 & 26 \\
& Santa Cruz & Yungay & 2868 & $\mathrm{~N} 2=70$ & 0.39 & 27 \\
Yungay & Yungay & Santa Cruz & 2463 & $\mathrm{~N} 3=125$ & 0.34 & 42 \\
Carhuaz & Pariahuanca & Pariahuanca & 2785 & $\mathrm{~N} 4=65$ & 0.2 & 13 \\
& Tinco & Tinco & 2581 & $\mathrm{~N} 5=58$ & 0.12 & 7 \\
& & Total & & $\mathrm{N}=448$ & & $\mathrm{n}=115$ \\
\hline
\end{tabular}

Tabla 2. Indicadores y subindicadores económicos, ambientales y sociales.

\begin{tabular}{|c|c|c|c|}
\hline \multicolumn{4}{|c|}{ Indicadores y Subindicadores Económicos de las unidades productivas de Amaranto } \\
\hline Indicador & Sub indicador & Clave & Unidad y forma de medida en campo \\
\hline \multicolumn{4}{|c|}{ Indicadores y subindicadores económicos } \\
\hline \multirow[t]{3}{*}{ A: Rentabilidad } & A1.- Superficie cultivada & SUCUT & Superficie cultivada (ha) \\
\hline & A2.- Rendimiento & RENDM & Rendimiento en $\mathrm{t} \mathrm{ha}^{-1}$ \\
\hline & A3.- Vías de acceso & VIASAC & Facilidad de acceso a parcelas y mercados \\
\hline B.- Ingreso económico & B1.- Ingreso Neto mensual & INGNM & Ingreso mensual (USD) \\
\hline \multirow[t]{3}{*}{ C: Riesgo económico } & C1.- Diversificación venta & DIVEN & Diversidad de productos agrícolas para la venta \\
\hline & C2.- Procesamiento & PROCE & Posibilidades de generar valor agregado \\
\hline & C3.- Dependencia de insumos & DEPEI & Dependencia de insumos agrícolas externos \\
\hline \multicolumn{4}{|c|}{ Indicadores y Subindicadores Ambientales } \\
\hline \multirow[t]{4}{*}{ A: Conservación del suelo } & A1.- Rotación de cultivos & ROTAC & Secuencia anual de instalación de cultivos \\
\hline & A2.- Diversificación de cultivos & DICUL & Número de cultivos por parcela \\
\hline & A3.- Tecnologías de cosecha & TECOS & Nivel de tecnificación en la parcela \\
\hline & A4.- Preparación de terreno & PRETE & Intensidad de labores de labranza de tierras \\
\hline \multirow[t]{2}{*}{ B.- Riesgo de erosión } & B1.- Pendiente predominante & PENTE & Declive del terreno, \\
\hline & B2.- Orientación de los surcos & ORSUR & Dirección de los surcos en las parcelas \\
\hline \multirow[t]{3}{*}{ C: Manejo de Biodiversidad } & C1.- Conservación de cultivares & COCNA & Conservación de cultivares nativos de Amaranto \\
\hline & C2.- Buenas prácticas agrícolas & BPRAG & Prácticas agrícolas en las unidades productivas \\
\hline & C3.- Manejo semilla de calidad & MASEM & Disponibilidad y acceso a semillas de semillas \\
\hline \multicolumn{4}{|c|}{ Indicadores y Subindicadores Sociales } \\
\hline \multirow{4}{*}{$\begin{array}{l}\text { A: Satisfacción necesidades } \\
\text { básicas }\end{array}$} & A1.- Vivienda & VIVIE & Tipo de vivienda del productor \\
\hline & A2.- Nivel de educación & EDUCA & Nivel de educación logrado por el productor \\
\hline & A3.- Servicios de salud & SALUD & Disponibilidad y acceso a servicios de salud \\
\hline & A4.- Servicios básicos & SEBAS & Acceso a servicios básicos de la vivienda \\
\hline \multirow[t]{2}{*}{ B: Sistema de producción } & B1.- Oferta tecnológica & OTECN & Acceso y uso de tecnologías de producción \\
\hline & B2.-Sistema de producción & SPROD & $\begin{array}{l}\text { El sistema de producción en la sostenibilidad de } \\
\text { los agroecosistemas }\end{array}$ \\
\hline C: Asociatividad & C1.- Nivel de integración social & NIVIS & Convivencia del productor en la comunidad \\
\hline D.- Nivel de satisfacción & D1.- Nivel de satisfacción del productor & NISAT & $\begin{array}{l}\text { Nivel de percepción de satisfacción del productor } \\
\text { con la producción de Amaranto }\end{array}$ \\
\hline
\end{tabular}

escala estandarizada (valor de juicio) que representa el valor de los indicadores con relación a la situación deseable (Silva y Ramírez 2017). Los datos de las encuestas se estandarizaron para cada indicador mediante transformación a una escala de 1 a 5 ; siendo 5 el mayor valor de sostenibilidad y 1 el más bajo (Pinedo et al. 2017, Pinedo et al. 2018). Los valores de cada indicador se expresaron en valores de la escala, para luego establecer ponderaciones de acuerdo con el grado de importancia y peso de cada indicador, para facilitar la detección de tendencias a nivel de sistema (Sarandón et al. 2006, Castelán et al. 2014, Silva y Ramírez 2017). Por lo que ningún valor de los indicadores debe ser menor al umbral mínimo establecido de 3 (Sarandón 2002). Los datos e información obtenidos se procesaron con el paquete estadístico SPSS.

\section{RESULTADOS Y DISCUSIÓN}

Para la sostenibilidad económica (Figura 1), la superficie cultivada (SUCUT) fue el subindicador que menos contribuyó a la rentabilidad de las unidades 


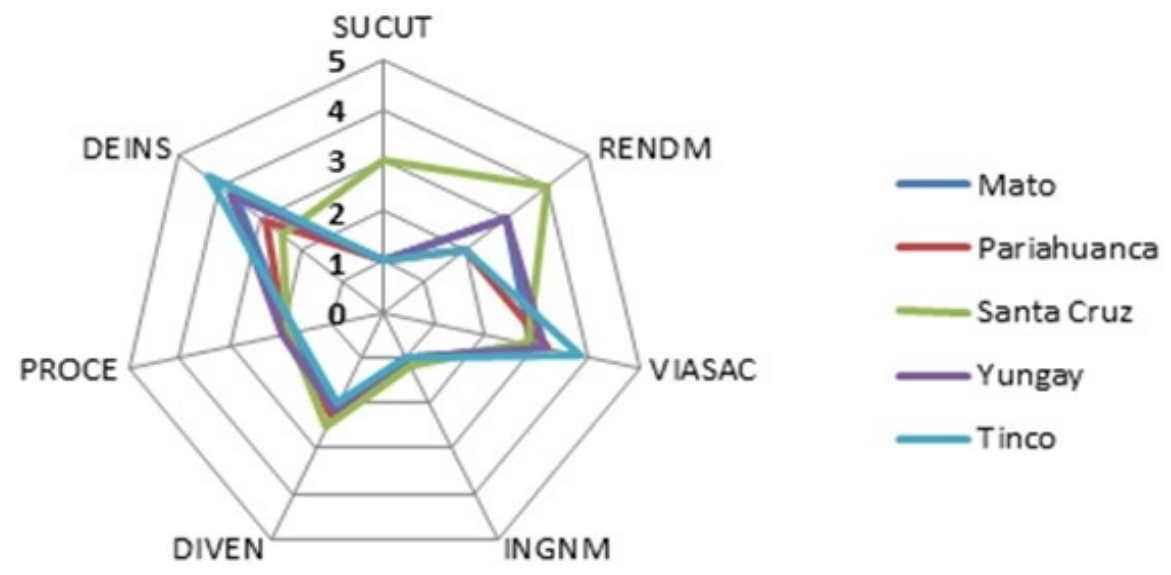

Figura 1. Niveles de sostenibilidad de los subindicadores económicos en las comunidades de Mato, Pariahuanca, Santa Cruz, Yungay y Tinco, del departamento de Ancash. SUCUT (Superficie Cultivada), PRODU (Productividad), VIACS (Vías de Acceso), INGNM (Ingreso Neto Mensual), DIVENT (Diversificación para la Venta), PROCES (Procesamiento), DEINS (Dependencia de insumos).

de producción de amaranto (UPA); solo en la comunidad de Santa Cruz tuvo el umbral mínimo de sostenibilidad (UMS) en las unidades de producción que tienen 1 ha de amaranto; mientras que en las comunidades de Tinco, Pariahuanca, Yungay y Mato el área de siembra fluctuó entre 0.10 y 0.50 ha, con orientación a las estrategias de seguridad alimentaria. Al respecto, Mejía y Gómez (2020) reportan que en el ámbito de estudio la producción de amaranto se realiza en pequeñas parcelas que no superan 1 ha. Mientras que Barreto et al. (2015) mencionan que en Ancash, predomina la agricultura de pequeña parcela o minifundista (27\%) con unidades productivas que fluctúan entre 1 y 2 ha. Mientras que el INEI (2013) y Maletta (2017) mencionan que la estructura productiva ha cambiado en los últimos 20 años, debido a la fragmentación de las unidades productivas; lo que dificulta el desarrollo de economías de escala, porque los recursos tierra y superficie cultivada se relacionan con la rentabilidad del cultivo (Mercado y Ubillus 2018, Pinedo et al. 2018).

El rendimiento (RENDM), indicador clave en la rentabilidad de las UPA, en las comunidades Mato, Santa Cruz y Yungay tuvo valores mayores al UMS con rendimiento entre 0.75 y $1.49 \mathrm{t} \mathrm{ha}^{-1}$; mientras que en Pariahuanca y Tinco los rendimientos fueron de 0.25 a $0.49 \mathrm{t} \mathrm{ha}^{-1}$, lo que las ubica como UPA en insostenibilidad. De acuerdo con MINAGRI (2018a) y
Mejía y Gómez (2020), el rendimiento promedio nacional es de $1885 \mathrm{~kg} \mathrm{ha}^{-1}$; mientras que en Ancash es de $1638 \mathrm{~kg} \mathrm{ha}^{-1}$ (MINAGRI 2017). Las vías de acceso (VIACS) a sus parcelas y los mercados son recursos estratégicos que puede mejorar la competitividad de la UPA. La comunidad de Santa Cruz se encuentra en desventaja frente a las otras comunidades por tener escasa infraestructura de carreteras, lo que la expone a mayor riesgo de deterioro de sus cosechas, y mayor dificultad para acceder a los mercados. Para el ingreso neto mensual (INGRNM), las cinco comunidades se encuentran en escala crítica de sostenibilidad, con ingresos entre 39.45 y 149.55 USDA, lo que las expone a condición de pobreza y vulnerabilidad de seguridad alimentaria. Debido a que el ingreso familiar es el pilar de una actividad productiva, se deben tener niveles aceptables de rentabilidad para ser sostenibles (Sarandón 2002, Sarandón y Nichols 2014, Vilches y Gil 2016). Pero uno de los retos más grandes que enfrentan los pequeños agricultores y en especial aquellos que utilizan especies subutilizadas son los altos costos en la comercialización de sus productos (Bosque et al. 2016, Bravo et al. 2010).

Para el riesgo económico, los subindicadores (DIVEN), y procesamiento de amaranto (PROCE) no alcanzan el UMS; por lo que poco contribuyen a la sostenibilidad. Por los cambios estructurales en 
tenencia de la tierra, superficie cultivada y lógica de producción tradicional, los sistemas predominantes son monocultivo, salvo algunas parcelas con policultivos (Mejía y Gómez 2020). En lo referente al subindicador dependencia de insumos (DEPEI) la comunidad de Tinco, tiene la mayor independencia de insumos externos; mientras que en la comunidad de Santa Cruz tiene alta dependencia, debido al uso de fertilizantes, plaguicidas, maquinaria agrícola y semillas mejoradas.

Para mejorar los niveles de sostenibilidad económica del amaranto, es necesario incluirse en el plan nacional de cultivos (MINAGRI, 2018b), ya que puede ser una alternativa importante por su alta demanda internacional, pero se requiere mejorar la tecnología de cultivo para incrementar el rendimiento y calidad. También, es necesario mejorar la articulación de la producción con los agentes y canales de comercialización para aprovechar los nichos de mercado; además de fortalecer la investigación y difusión de tecnologías para el manejo del cultivo (Sánchez et al. 2016)

Para el indicador conservación del suelo, el subindicador rotación de cultivos (ROTAC) para la comunidad de Santa Cruz presentó el mayor nivel de aporte a la sostenibilidad ambiental, debido a que los productores rotan el cultivo de amaranto cada dos años con siembra intermedia de fabáceas como haba (Vicia faba), arveja (Pisum sativum) o tarwi (Lupinus mutabilis); mientras que en Mato y Yungay esta práctica se realiza previo a una rotación de cultivo de arveja para volver a sembrar el mismo año; en tanto que en Pariahuanca y Tinco la rotación se realiza después de una siembra de cereales (maíz o trigo). Con respecto, a tecnología de cosecha (TECOS), los productores no cuentan con maquinarias equipos 0 herramientas que faciliten las labores de cosecha, por lo que usualmente realizan la cosecha con palos y garrotes para desprender los granos. Esta práctica si bien es tradicional puede ocasionar hasta un $30 \%$ de pérdidas. Con respecto, a la preparación de terreno (PRETE), la comunidad de Pariahuanca realiza la preparación del terreno con desterronado y labranza manual con lo que alcanza el UMS, mientras que las otras comunidades tienen valores entre
2.19 y 2.71 , lo que cual indica que no aportan a la sostenibilidad por realizar la preparación del terrono con yunta $o$ arado de buey (Figura 2).

Para el indicador riesgo de erosión, la pendiente predominante (PENTE) en Mato y Santa Cruz tiene menor riesgo de erosión, con valores entre 16 y $25 \%$; mientras que en Pariahuanca Yungay y Tinco la pendiente alcanza hasta $35 \%$, lo que afecta la conservación de suelos por el mayor riesgo de erosión. Otro factor, que perjudica en la estabilidad y fertilidad de los suelos es la orientación de los surcos (ORSUR), en Mato y Santa Cruz se tienen prácticas conservacionistas al realizar la siembra de acuerdo con las curvas de nivel de las parcelas; mientras que en Pariahuanca, Santa Cruz y Tinco la orientación de los surcos es a favor de la pendiente. Al respecto, el MINAGRI (2016b) reporta que el $99 \%$ de la superficie cultivada en el Perú presenta problemas de erosión de leve a severo.

En el indicador manejo de la biodiversidad, en la conservación de cultivares nativos (COGNA), Santa Cruz conserva y usa hasta 3 variedades entre mejoradas y nativas; mientras que el resto de las comunidades hasta dos variedades. Pero predomina la siembra de parcelas de amaranto con una sola variedad, al respecto Mejía y Gómez (2020) reporta la introducción de las variedades mejoradas Oscar Blanco y Centenario, y del cultivar nativo el Caracino. Como consecuencia de la introducción de variedades mejoradas y las prácticas de monocultivo el proceso de desplazamiento de variedades nativas de alto valor alimenticio, genético y cultural es evidente (Sarandón y Flores 2014, Ponce et al. 2015, Pinedo et al. 2018). Ya que las prácticas de monocultivo no solo afectan la conservación de la agrobiodiversidad, sino también el equilibrio biológico con la consecuente aparición de plagas y enfermedades en los cultivos (UN/CEPAL/FAO/IICA 2017). En cambio, los sistemas de policultivos tienen repercusiones positivas en el mantenimiento de los suelos y regulación natural de las plagas (Vallejo et al. 2018).

Para la aplicación de buenas prácticas agrícolas (BPRAG) se observaron bajos niveles, ya que no cuentan con sistemas de recojo y almacenamiento de envases de residuos agrícolas, los cuales son 


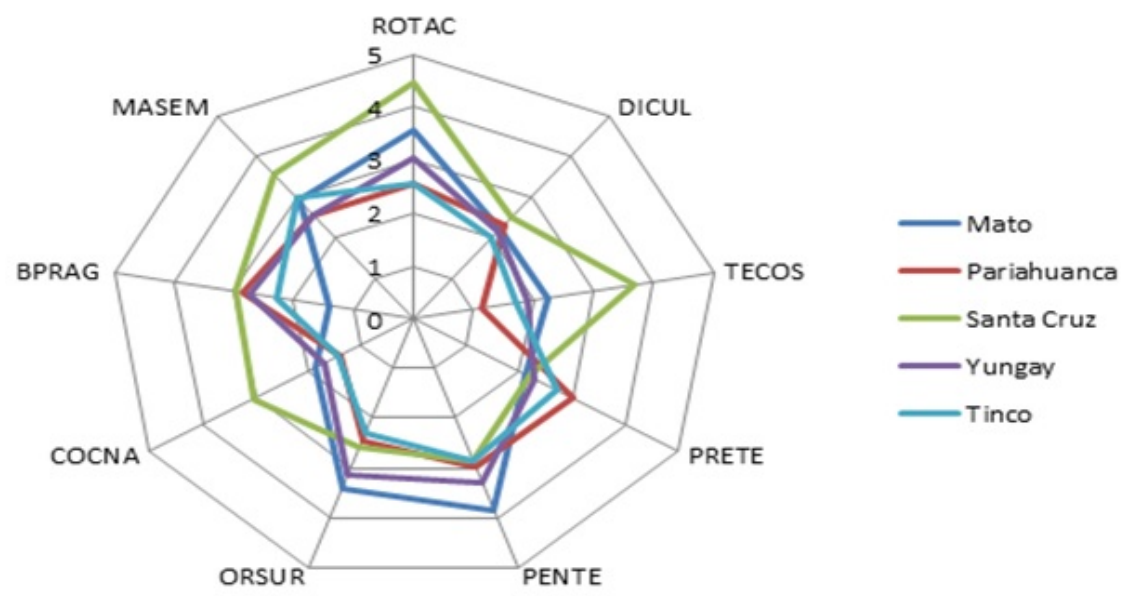

Figura 2. Valor de los subindicadores ambientales en las comunidades de Mato, Pariahuanca, Santa cruz Yungay y Tinco, del departamento de Ancash. ROTAC (Rotación de cultivos), DICUL (Diversificación de cultivos), TECOS (Tecnología de cosecha), PRETE (Preparación de terreno), PENTE (Pendiente predominante), ORSUR (Orientación de surcos), COCNA (Conservación de cultivares nativos) BPRAG (Buenas Prácticas Agrícolas), MASEM (Manejo de Semillas).

abandonados en la parcela; mientras que en comunidades como Tinco se acostumbra quemar los envases. La falta de acompañamiento de programas de fomento al cultivo de amaranto y la pérdida gradual de manejo tradicional del cultivo puede generar impactos ambientales negativos, si no se promueve la producción sostenible de cultivos (Pinedo 2019).

Para el manejo de semilla (MASEM) en Santa Cruz se utiliza semilla de origen conocido y certificado, lo que aporta en la sostenibilidad del sistema, mientras que en las otras comunidades se utiliza semilla de origen desconocido y de baja calidad, lo que repercute en la rentabilidad. Al respecto, Pinedo (2019) reporta que el uso de semilla de calidad en granos andinos como la quinua puede incrementar el rendimiento hasta un $56 \%$. Pero la tasa de uso de semilla certificada en la sierra del Perú es baja, lo que se refleja en la baja productividad de los cultivos (MINAGRI 2015, Silva y Ramírez 2017). Asimismo, es necesario tener en cuenta el potencial de semillas locales como la variedad Caracino, originario de Ancash, que puede alcanzar rendimientos similares e incluso superiores a las variedades Oscar Blanco y Centenario (Mejía y Gómez 2020). Frente a esta situación de desinterés de programas de mejoramiento de variedades locales de alto valor genético, los agricultores recurren a un pequeño número de variedades para responder a las normas de calidad exigidas por las empresas agroindustriales (Dufumier 2014). Las variedades locales, al desarrollarse en las áreas altoandinas se adapta a diversos entornos agroclimáticos, por lo que tienen la ventaja de haber sido seleccionadas para resistir las condiciones de estrés, lo que contribuye a la producción sostenible, y por tanto a la estabilidad de los agroecosistemas (Bravo et al. 2010, Vera y Lerma 2015). Pero la sostenibilidad ambiental depende también de otros factores relacionados como los niveles de ingresos económicos y acceso a servicios de vivienda, educación y salud (Altieri y Nichols 2000, Pinedo 2019).

Para la sostenibilidad social (Figura 3), se observa que los indicadores de menor aporte a la sostenibilidad de las UPA fueron la oferta tecnológica (OTECN), educación (EDUCA) y la asistencia técnica, mientras que los subindicadores vivienda (VIVIE) y servicios básicos (SEBAS) contribuyen a mejorar la calidad de vida de los agricultores, lo que indica que en estas comunidades cuentan con viviendas convencionales (construcción de concreto con tabiques y vigas de albañilería), tienen educación secundaria o técnica, cuentan con servicios básicos como agua, luz, electricidad y desagüe, están satisfechos con su sistemas de producción y el nivel de organización e integración se encuentran en rango 


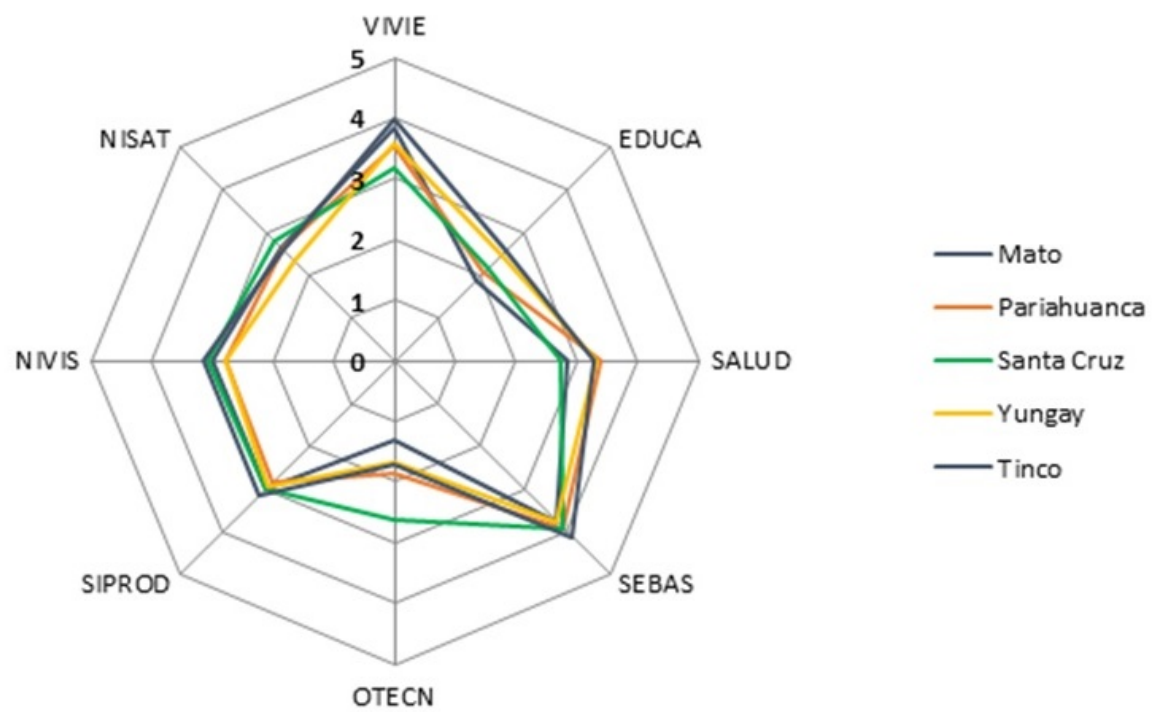

Figura 3. Valores de los subindicadores de la dimensión social en las comunidades de Mato, Pariahuanca, Santa Cruz, Yungay y Tinco. VIVIE (Vivienda), EDUCA (Educación), SALUD (Salud), SEBAS (Servicios básicos) SIPROD (Sistema de producción), NIVIS (Nivel de integración social), NISAT (Asistencia Técnica y Capacitación).

aceptable. En tanto que la EDUCA refleja la realidad de la zona, al respecto el Gobierno Regional de Ancash (2008), reportan que solo el $27 \%$ de la población rural de Ancash tiene algún nivel de educación superior, con niveles de analfabetismo del $28 \%$ en mujeres y $9 \%$ en hombres. La realidad de la OTECN y la asistencia técnica en la zona de estudio se refleja en los resultados del cuarto censo nacional agropecuario, que reporta 2160000 productores, de los cuales el $90 \%$ son pequeños productores y solo el $10 \%$ reciben algún tipo de capacitación o asistencia técnica (INEI 2013). La escasa oferta tecnológica es mayor en cultivos como el amaranto; debido a que la innovación es una de las debilidades que el país tiene para ser competitivo (MINAGRI 2016), como lo reconoce el Reporte Anual de Competitividad Global 2014-2015 del Foro Económico Mundial (Schwab 2014).

La producción de maranto en Mato, Pariahuanca y Yungay no fueron sostenibles en ninguna de las dimensiones evaluadas, con valores menores de 3 , mientras que el índice de sostenibilidad general (ISG) osciló entre 2.35 y 2.84 (Figura 4). Para ser sostenible de acuerdo con la escala de valoración establecida, los valores deben ser mayores de 3 (Pinedo et al. 2018, Sarandón y
Nichols 2014). Al respecto Mejía y Gómez (2020) en una evaluación de sostenibilidad realizada en Mato, Yungay y Santa Cruz reportan valores de 2.52, 2.54 y 2.93 de ISG, los cuales son similares a los del presente estudio. Lo que confirma que la producción de amaranto en Ancash no es rentable y no contribuye a mejorar las condiciones y medios de vida. Para ser sostenibles las UPA requieren mejorar en términos económicos, ambientales, sociales e institucionales (FAO 2014), además de políticas favorables y tecnología (Maletta 2017, UN/CEPAL/FAO/IICA 2017). Pero para el Gobierno regional de Ancash (2008), el amaranto no figura en las estadísticas, lo cual muestra el escaso interés en promocionar su cultivo. Por lo que es necesario no solo incrementar las áreas de cultivo, sino que también se requiere fomentar mejores prácticas de cultivo y generación de valor agregado.

Actualmente el amaranto constituye una buena alternativa alimenticia y económica (Vera y Lerma 2015). Pero en el caso peruano a pesar de que el objetivo general de la política agraria del MINAGRI (2016), es contribuir a la seguridad alimentaria y nutricional de los agricultores, mejorando sus ingresos económicos y medios de vida, el cultivo del 


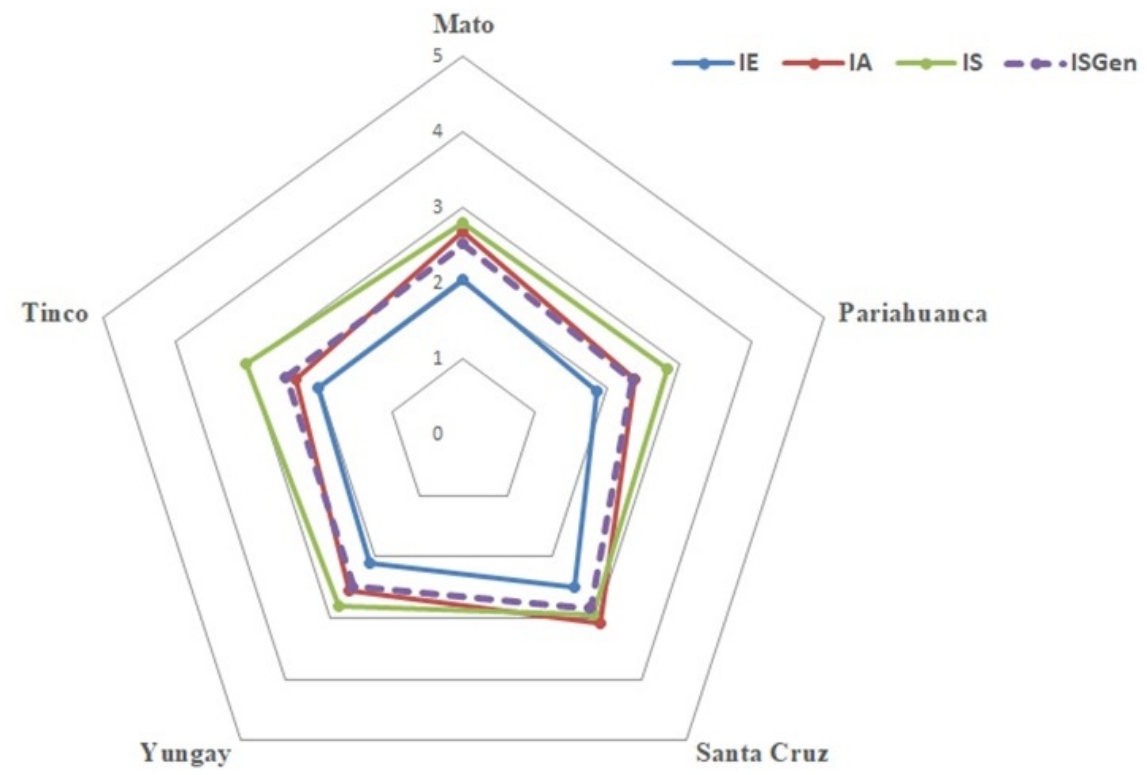

Figura 4. Valor de los Indicadores Económicos (IK), Indicador Ambiental (IA), Indicadores Sociales (IS) e Índice de Sostenibilidad General (ISG) de la producción de Amaranto, en las comunidades de Mato, Yungay y Santa Cruz, Ancash.

amaranto está excluido, debido a que no aparece como cultivos de seguridad alimentaria en el Plan Nacional de Cultivos (MINAGRI 2018b). La ausencia de políticas de fomento, los factores climáticos adversos y la migración de los productores de las zonas rurales a las ciudades, inciden en la insostenibilidad económica del cultivo, por lo que los productores han cambiado de cultivo o abandonado la actividad agrícola (Sánchez et al. 2016).

El cultivo de amaranto, no solo debe verse como refugio de pequeños productores o productores de subsistencia (Maletta 2017, Ponce et al. 2015). Además de que las habilidades y las prácticas autóctonas, mantenidas y mejoradas de generación en generación, no deben dejarse de lado, debido a que proporcionan medios de subsistencia (Hernández et al. 2018, Pinedo 2019). Al respecto, Pinedo (2019) menciona que el conocimiento tradicional adquiere relevancia en el manejo de la biodiversidad y en el desarrollo de adecuadas técnicas de producción. La intensificación del cultivo y el monocultivo, no son la solución; debido a que pueden ocasionar graves daños ambientales (Altieri y Nichols 2000, Dufumier 2014, FAO 2014, Vallejo et al. 2018). Por lo que las propuestas deben orientarse a la elaboración de políticas dirigidas a incrementar la productividad, reducir la inequidad en las cadenas agroalimentarias, fortalecer la resiliencia y reducción del impacto ambiental de los sistemas productivos (FAO/CEPAL/IICA 2017).

Las unidades agrícolas en las comunidades evaluadas tienen indicadores económicos, ambientales y sociales debajo del umbral mínimo aceptable, baja generación de ingresos, pero conservan los recursos y la biodiversidad. El índice de sostenibilidad general fue no sostenible, por lo que los productores realizan otras actividades complementarias para mejorar sus ingresos económicos.

\section{LITERATURA CITADA}

Altieri M, Nicholls C (2000) Agroecología. Teoría y práctica para una agricultura sustentable. Primera Edición. PNUMA. México. 250p.

Barreto J, Julca A, Canto M (2015) Sostenibilidad ecológica de la producción agropecuaria tradicional de Carhuaz, 
Ancash, Perú. Aporte Santiaguino 8: 219-228.

Bosque H, Trigo R, Mercado G, Rojas V, Cestari M, Montes de Oca M, Delgadillo C (2016) Revalorización sostenible de la agrobiodiversidad a través de la gastronomía: la visión del proyecto LATINCROP. Revista de Investigación e Innovación Agropecuaria y de Recursos Naturales 3: 214-217.

Bravo R, Valdivia R, Andrade K, Padulosi S, Jäger M (2010) Granos Andinos. Avances, logros y experiencias desarrolladas en quinua, cañihua y Amaranto en Perú. Bioversity International. Roma, Italia. 135p.

Castelán R, Tamaríz V, Ruiz J, Linares G (2014) Evaluación de la sustentabilidad de la actividad agrícola de tres localidades campesinas en Pahuatlán, Puebla. Ecosistemas y Recursos Agropecuarios 1: 219-231.

Dufumier M (2014) Agriculturas familiares, fertilidad de los suelos y sostenibilidad de los agroecosistemas. En: Francisco HF, François H, Pilar LA (Ed.) Agriculturas campesinas en Latinoamérica: propuestas y desafíos. Editorial IAEN. Quito, Ecuador. pp: 55-64.

FAO (2014) Building a common vision for sustainable food and agriculture. Principles and approaches. Organización de las Naciones Unidas para la Alimentación y la Agricultura. Roma, Italia. 50p.

Gobierno Regional de Ancash (2008) Plan de Desarrollo Regional Concertado 2008-2021 de Ancash. Gerencia de Planeamiento. Gobierno Regional de Ancash. Perú. 125p.

Hernández BR, Santiago DP, Miguel A E, Cruz C, Maldonado RJ (2018) Empresas sociales rurales, estrategia de desarrollo sustentable y conservación del patrimonio cultural inmaterial. Caso: amaranto (Amaranthus spp) de Mesoamérica. Revista Mexicana de Agronegocios 42: 955-967.

INEI (2013) Resultados definitivos. IV Censo Nacional Agropecuario 2012. Instituto Nacional de Estadística e Informática. Lima, Perú. 62p.

Maletta H (2017) La pequeña agricultura familiar en el Perú. Una tipología microrregionalizada. En IV Censo Nacional Agropecuario 2012: Investigaciones para la toma de decisiones en políticas públicas. Libro V. FAO. Lima, Perú. 208p.

Matías G, Hernández BR, Peña V, Torres NG, Espinoza VA, Ramírez L (2018) Usos actuales y potenciales del Amaranto (Amaranthus spp.). Journal of Negative and No Positive Results 3: 423-436.

Mejia R, Gomez L (2020) Sostenibilidad de las unidades de producción del cultivo de Amaranto (Amaranthus caudatus) en las provincias de Yungay-Huaylas, región Ancash. Revista Ciencia e Investigación 5: 80-90.

Mercado W, Ubillus K (2017) Characterization of producers and quinoa supply chains in the Peruvian regions of Puno and Junin. Scientia Agropecuaria 8: 251-265.

MINAGRI (2015) Estrategia nacional de agricultura familiar 2015-2021. Ministerio de Agricultura y Riego del Perú. República del Lima, Perú. 123p.

MINAGRI (2016) Política Nacional Agraria. Ministerio de Agricultura y Riego del Perú. Ministerio de Agricultura y Riego. República del Perú. 63p. Fecha de consulta 20 de mayo de 2020.

MINAGRI (2017) Anuario estadístico de producción agrícola y ganadera 2016. Ministerio de Agricultura y Riego del Perú. Sistema Integrado de Estadística Agraria. Perú. 155p.

MINAGRI (2018a) Anuario estadístico de la producción agrícola 2017. Sistema Integrado de Estadística Agraria. Ministerio de Agricultura y Riego del Perú. Perú. 370p.

MINAGRI (2018b) Plan Nacional de Cultivos. Campaña agrícola 2018-2019. Ministerio de Agricultura y Riego del Perú. Lima, Perú. 293p. 
Pinedo R (2019) Innovaciones tecnológicas con metodología de ECA en producción y adopción de uso de semilla certificada en sistemas de Agricultura Familiar. Tierra Nuestra 13: 77-86.

Pinedo R, Gómez L, Julca O (2017) Indicadores de sostenibilidad de sistemas de producción de quinua en Chiara, Ayacucho. Aporte Santiaguino 10: 197-210.

Pinedo R, Gómez L, Julca O (2018) Sostenibilidad de sistemas de producción de quinua (Chenopodium quinoa Willd.). Ecosistemas y Recursos Agropecuarios 5: 399-409.

Ponce C, Arnillas CA, Escobal J (2015) Cambio climático, uso de riego y estrategias de diversificación de cultivos en la sierra peruana. En: Escobal J, Ricardo F, Eduardo Z (ed). Agricultura peruana: nuevas miradas desde el Censo Agropecuario. Lima, Perú. pp. 171-217.

Rocha C, Mora J, Romero J (2016) Tipología de sistemas de producción en la zona rural del municipio de lbagué, Colombia. Agronomía Mesoamericana 27: 253-264.

Sánchez J, Argumedo A, Álvarez J F, Méndez J A, Ortiz B (2016) Análisis económico del sistema socio técnico del cultivo de amaranto en Tochimilco, Puebla. Acta Universitaria 26: 95-104.

Sarandón SJ (2002) El desarrollo y uso de indicadores para evaluar la sustentabilidad de los agroecosistemas. En: Sarandón SJ (ed). Agroecologia: El camino para una agricultura sustentable. Ediciones Científicas Americanas. La Plata, Argentina. pp: 393-414.

Sarandón SJ, Flores C (2014) Agroecología: bases teóricas para el diseño y manejo de Agroecosistemas sustentables. Primera Edición. Universidad Nacional de La Plata. Argentina. 466p.

Schwab K (2014) The global competitiveness report 2014-2015. World Economic Forum. Geneva, Switzerland. $548 \mathrm{p}$.

Sarandón SJ, Zuluaga M, Cieza R, Gómez C, Janjetic L, Negrete E (2006) Evaluación de la sustentabilidad de sistemas agrícolas en fincas de Misiones, Argentina, mediante el uso de indicadores. Agroecología 1: 19-28.

Schwab K (2014) The global competitiveness report 2014-2015. World Economic Forum. Geneva, Switzerland. 548 .

Silva L, Ramírez O (2017) Evaluación de agroecosistemas mediante indicadores de sostenibilidad en San José de las Lajas, provincia de Mayabeque, Cuba. Luna Azul 44: 120-152.

UN/CEPAL/FAO/IICA (2017) Perspectivas de la agricultura y del desarrollo rural en las Américas: una mirada hacia América Latina y el Caribe 2017-2018. Comunidad Económica para América Latina/Programa de las Naciones Unidas para la Alimentación y la Agricultura/Instituto Interamericano de Cooperación para la Agricultura. San José, Costa Rica. 217p.

Vallejo VE, Afanador LN, Hernández MA, Parra DC (2018) Efecto de la implementación de diferentes sistemas agrícolas sobre la calidad del suelo en el municipio de Cachipay, Cundinamarca, Colombia. Bioagro 30: 27-38.

Vera A, Lerma DL (2015) Estrategia de comercialización competitiva del amaranto en San Luis Potosí. Perspectivas 10: 71-93.

Vilches A, Gil D (2016) La transición a la Sostenibilidad como objetivo urgente para la superación de la crisis sistémica actual. Revista Eureka sobre Enseñanza y Divulgación de las Ciencias 13: 395-407. 